\title{
Hacia una ética de liberación para la traducción bíblica
}

\author{
Toward a liberation ethics for biblical translation
}

\section{Esteban Voth ${ }^{*}$}

Hebrew Union College, Jerusalém, Israel

\section{Resúmen}

El presente estudio intenta analizar el tema de ética y la traducción bíblica, al ver cuidadosamente situaciones específicas tomadas de la vida real. Dentro de la metodología de estudio de casos, se abordan dos realidades principales: ideología y mercadotecnia. El estudio ahonda en las presiones e influencias que estas realidades ejercen sobre el proceso de la traducción bíblica. La traducción bíblica nunca está totalmente libre de estas dos presiones inminentes y por lo tanto la ética de la traducción bíblica afectada por ambas. Como meta final, el estudio busca proponer una ética de traducción bíblica que sea flexible pero, a la vez, liberadora. Lo que aqui se propone es que la necesidad humana debe ser colocada al frente y al centro del desarrollo y articulación de toda ética de la traducción bíblica.

Palabras-clave: Traducción bíblica. Ética. Ideologia. Biblia. Interpretación. 
VOTH, E.

\section{Abstract}

The present study aims to analise the bond between Ethics and biblical translation, examining acurately specific cases taken from real life. In according to the methodology of case studies, this approach has two focuses: ideology and market-tecnique. Author examines the pressions and influences of both realities, ideology and market-tecnique, over biblical translation process. Indeed, biblical translation is never totaly free from these two imminent influences, affecting the Ethics in biblical translation task. Seeking for a good method, Author suggests an Ethics to biblical translation that may be both, flexible and liberator. The human needs must have priority for all elaboration and articulation of the Ethics in biblical translation work.

Keywords: Biblical translation. Ethics. Ideology. Bible. Interpretation.

\section{Introducción}

La Biblia es considerada por muchos en el mundo occidental como el libro más importante de todos. Quizás uno podría decir que ningún otro libro o colección de escritos ha sido traducido más y con mayor cuidado y a más idiomas que la Biblia. Esto por supuesto ha generado una miríada de opiniones, no siendo la menor de ellas la frase repetida de traduttore traditore. Consecuentemente, inmediatamente surge la pregunta: «¿Traidor a qué, a quién? » ¿A quiénes o qué estamos traicionando nosotros como traductores? Esta realidad se complica aún más por la convincente insinuación de que la traducción es verdaderamente imposible, sin dejar de mencionar la imposibilidad de la traducción bíblica. El rabino Simlai afirmó una vez que la traducción es una tarea imposible: «El que traduce es un hereje, pero el que se rehúsa a traducir es un blasfemo». Si esto es verdad, debemos proponer que en cuanto a la práctica y profesión de la traducción, "palo si bogas, palo si no».

Walter Brueggemann (1997, p. 180) ha acuñado la frase «Textos que perduran, palabras que explotan» para referirse al proceso tradicionante que está presente en la Biblia, en especial con relación a los profetas. 
Él sugiere que en ciertos momentos en la historia de la apropriación del texto, por parte de la comunidade de fe, algo nuevo ocurre

Lo que ha sido tradición, cerniéndose de manera latente, se manifiesta ahora en la experiencia. En el momento de hablar y escuchar, la tradición atesorada se convierte en una experiencia presente, inimitable, sin parangón, irreversible. En esa pronunciación, la palabra sí conduce a la realidade (BRUEGGEMANN, 1997, p. 181).

Si en efecto Brueggeman tiene razón, y creemos que así es, la traducción de la Biblia no solo es un ejercicio difícil, sino que el desarrollo de una traducción ética para la traducción bíblica se convierte en una hazaña muy compleja. De hecho, vamos a sugerir desde un principio, que una ética definitiva e integral de la traducción bíblica es una imposibilidad. Y sin embargo, la búsqueda por una ética, en vez de la ética, es a nuestro juicio una tarea digna de realizar.

Nuestra meta no es en este momento discutir los muchos y diversos problemas que el traductor bíblico enfrenta. Nuestra meta principal aquí es explorar varios temas que son pertinentes al desarrollo de la ética de la traducción bíblica. Ya debe de ser evidente para todo lector que este autor considera que cualquier marco teórico considerado en este ejercicio es por naturaleza extremadamente provisional. En principio, nuestra metodología será dialógica. Llevaremos a cabo un diálogo con Towner, Pym, Chesterman, Lyotard y Dussel, además de Wittgenstein y Spinoza (cf. DELEUZE, 2004; YENGOYAN, 2003). Este diálogo se enfocará en dos temas significativos: ideología y mercadotecnia.

La discusión en torno a estos temas será ilustrada por ejemplos reales provenientes de proyectos de traducción en las Américas. Ninguno de estos ejemplos o situaciones que serán presentados son hipotéticos o ficticios. En cambio, son concretos, reales, y uno podría posiblemente considerarlos como históricos. Algunos de estos ejemplos servirán como estudios que pueden ayudar a agudizar ciertas cuestiones éticas que en definitiva son importantes en la traducción bíblica.

Después de considerar estos dos temas principales que se relacionan con el desarrollo de una posible ética de la traducción bíblica, intentaremos trabajar mediante una propuesta que propondrá algunas 
alternativas y pautas para la articulación de un modelo flexible de ética de traducción bíblica. La razón de que un énfasis se ponga en lo flexible es porque consideramos que la cultura en sus varias formas y expresiones mitiga cualquier intento de desarrollar un modelo rígido, fijo y cerrado. Si la cultura como un conjunto de categorías realizadas o estructuras, concretas y conscientes, brinda estilos de vida y significados a una sociedad en particular (cf. YENGOYAN, 2003), entonces cualquier ética debe ser lo suficientemente flexible para abarcar esta realidad compleja.

\section{Ideología y traducción bíblica}

Hemos escrito en otros estudios que ninguna traducción de cualquier texto es verdaderamente neutral u objetiva. Con esto queremos decir que las traducciones de textos nunca toman lugar en un vacío. Son producidas en lugares específicos, en momentos específicos, bajo condiciones específicas. Esto significa que un número de factores se llevan a cabo en el ejercicio de la traducción. Entre estos factores, sugiero que los más críticos son las realidades de raza, clase, género, historias personales, persuasiones teológicas, alianzas políticas, distinciones culturales y, por último - pero no por eso menos importante —, cuestiones de mercadotecnia (cf. VOTH, 2003).

Todos estos factores contribuyen a la ideología de cualquier traductor o equipo de traductores. Y sin embargo, ¿a qué nos referimos en realidad por ideología? En pocas palabras, ideología se puede referir a ese cuerpo sistemático de conceptos que existen, caracterizan y definen la vida y cultura humana. En un sentido, puede ser comparada a «cosmovisión». Tiene que ver con la manera en que un individuo o grupo entiende y define la realidad.

Para los fines de este estudio, vamos a hacer una distinción entre cosmovisión e ideología. La diferencia principal se basa en la suposición de que cualquier persona está más consciente de su ideología que la de su cosmovisión. Ideología es muchas veces algo que uno elige y que por consecuencia está mucho más permeado por la subjetividad y el interés. Hay muchos tipos de ideologías asumidas por individuos y grupos. Podemos 
hablar de ideologías políticas, religiosas, epistemológicas, económicas, sociales, por nombrar unas cuantas. Estas ideologías no son nunca inocentes o neutrales, pero siempre están presentes. Por lo tanto, ninguna traducción de cualquier texto es inocente o neutral. No existe tal cosa como una "traducción inmaculada". Esta realidad, la cual admitimos, ha sido enunciada en términos algo contundentes, e inevitablemente se manifiesta en cualquier discusión sobre una ética para la traducción y en particular para la traducción bíblica. Consideramos que Stanley Porter está en lo correcto cuando observa que: "La historia de la traducción bíblica está cargada con temas ideológicos” (PORTER, 1999, p. 18).

Es por esta razón que sugerimos que la articulación de una ética de traducción bíblica, debe tomar en consideración la constante e inexorable presencia de la ideología. Si una ética de la traducción bíblica no lucha con esta realidad, o elige ignorarla por completo, será una ética carente de credibilidad. Reconocemos al principio que esto no es una tarea fácil, ni tampoco es un asunto que debemos resolver. A lo sumo podemos decir que estamos en el proceso de asumir la realidad de la ideología en el ejercicio de la articulación de una ética. Las respuestas finales y definitivas no están en el horizonte inmediato. Dada la situación, procederemos a considerar algunos ejemplos actuales o estudios de caso que ilustrarán y brindarán elementos que deben de ser evaluados.

\section{Ideología: estudio de caso}

Al discutir y describir estos casos, cambiaré a la primera persona.

\section{Ejemplo 1:}

En 1990 comencé a trabajar en una nueva traducción para la Sociedad Bíblica Internacional. Fui elegido a ser el líder de equipo de Antiguo Testamento. Esta sería una traducción de las lenguas originales al español. Las pautas exegéticas, estilísticas y de formatos serían las mismas seguidas por el equipo que produjo la Nueva Versión Internacional para el idioma inglés. En términos de criterios de traducción, era intentar 
y forjar un camino intermedio entre una traducción literal y una traducción totalmente de equivalencia funcional. Trabajamos a partir de cuatro máximas: precisión, belleza, claridad y dignidad.

Poco después de que la traducción iniciara, se llevó a cabo un debate con respecto al tema del uso de las mayúsculas. El español es una lengua que es bastante tacaña en lo que respecta el uso de letras mayúsculas. Los títulos de libros, artículos, etc, solo escriben en mayúscula la primera letra de la primera palabra. Los nombres de los idiomas, meses del año y días de la semana no se escriben en mayúscula. En el proceso de elaborar la Nueva Versión Internacional, surgió el tema de si se debía escribir en mayúscula la palabra «espíritu» en el Antiguo Testamento, sobre todo cuando claramente se refería al espíritu de Dios. Hubo algunas cuestiones de mercadotecnia que surgieron, pero no fueron las más contundentes.

La discusión se polarizó porque el equipo de Nuevo Testamento argumentó a favor de escribir la palabra «espíritu» en mayúscula, mientras que el equipo de Antiguo Testamento unánimemente prefirió dejar la palabra sin mayúsculas. Muy pronto en la discusión, los factores ideológicos entraron en juego. Uno de los más importantes fue la presuposición de que el Antiguo Testamento necesita ser interpretada a la luz del Nuevo. Esto significa que uno lee, interpreta y traduce el Antiguo Testamento con los ojos del Nuevo Testamento.

Por otra parte, el equipo de Antiguo Testamento, el cual yo representaba, argumentaba que era incorrecto leer en los contextos del Antiguo Testamento el concepto del Nuevo Testamento acerca del Espíritu Santo, mismo que había suscitado escribir en mayúscula la palabra "espíritu». Un contexto muy crítico que generó mucho debate fue Génesis 1.2 lo cual desde luego brinda otras alternativas. La discusión se mantuvo por varios años. Eventualmente, la ideología del Nuevo Testamento prevaleció. La decisión final no se basó en una exégesis cuidadosa de un texto antiguo. Fue el resultado de una interpretación basada claramente en una ideología. Un voto final se tomó incluyendo a todos los traductores. Como habían más traductores en el equipo de Nuevo Testamento que en el de su contraparte, la palabra «espíritu» se escribió en mayúscula a lo largo de casi todo el Antiguo Testamento de la NVI. 
¿Fue esta una decisión ética correcta? O quizás debamos de preguntarnos, ¿se siguió un proceso éticamente correcto? ¿Cuáles factores influyeron en la decisión y el resultado final del debate? Ciertamente el factor ideológico fue uno importante. Sin embargo, uno no puede descartar la cuestión de mercadotecnia (la cual será discutida posteriormente). Fue bastante evidente que los miembros del equipo de traducción del Nuevo Testamento estaban mucho más preocupados por las posibles reacciones negativas que podrían ser provocadas al no escribir en mayúscula la palabra «espíritu». Hablaremos más al respecto posteriormente, conjuntamente con la Reina Valera 1995. Pero la pregunta se mantiene: ¿cuáles criterios éticos deben de ser invocados en este tipo de situación?

Quizás debamos de reconocer que tenemos cuestiones de lealtad dividida en un caso como este. De hecho, parece que muchas lealtades entran en juego: lealtad al Antiguo Testamente, lealtad al Nuevo Testamento, lealtad a la tradición de la traducción, lealtad a la sociedad que auspicia el trabajo, y lealtad al consumidor, entre otros. Pym ha destacado correctamente que los traductores rara vez están por encima de la sospecha (cf. PYM, 1992). Yo diría que los traductores bíblicos nunca están por encima de la sospecha y las decisiones como la que acabo de describir van a generar aún más sospecha sobre todo entre ciertas comunidades de fe. Así que, ¿existen reglas que se puedan seguir aquí? En mi propio caso personal, ¿tiene prioridad la ética profesional sobre la ética personal en este punto? Ya debe ser obvio que tengo más preguntas que respuestas.

\section{Ejemplo 2:}

En el mismo proyecto de traducción de la NVI surgió otra cuestión candente. En traducciones más tradicionales e históricas en español, Juan 1.1 dice: En el principio era el Verbo. Cuando Casiodoro de Reina tradujo logos, empleó «palabra». Sin embargo, en una de las revisiones posteriores hecha al texto, la palabra "verbo» se introdujo dentro del contexto. Por siglos esto se consideró como la traducción aceptada tanto en los círculos católicos como protestantes. No obstante, en el siglo XX, muchas nuevas traducciones como Dios Habla Hoy, El libro del pueblo de Dios, Cantera 
Iglesias y otros decidieron traducir logos como «palabra». Ahora bien, se debe reconocer que la tradición está tan firmemente afianzada que cuando uno busca la palabra «verbo» en todos los diccionarios prestigiosos de la lengua española, los cuales provienen de la Real Academia Española, uno encuentra como la primera acepción para verbo: segunda persona de la Santísima Trinidad. Muchos años antes del proyecto de la traducción de la NVI, los estudiosos llegaron a la conclusión de que la traducción más precisa y preferible para logos era palabra. Por ende, la traducción más lógica y exegéticamente precisa de logos para Juan 1.1 hubiera sido palabra.

Sin embargo, una vez más los asuntos ideológicos y la tradición entraron en juego. La palabra verbo lleva una carga teológica y espiritual tan grande que se hace muy difícil para los traductores cambiarla. Por consiguiente, después de todas las discusiones y los debates, cuando se sometió a votación el asunto, la tradición prevaleció. Mientras que la NVI se enorgullece de basarse en la erudición más reciente y actual, en este momento ha cedido a la tradición y las presiones ideológicas. Las cuestiones éticas surgen una vez más.

Pym (cf. 1992, p. 69) habla bastante acerca del trabajo en equipo. Él aboga por una prohibición de soledad. Estoy muy de acuerdo, y sin embargo en los casos recién discutidos el trabajo en equipo no ayudó en liberar el proceso de traducción de las presiones y el condicionamiento de la ideología. Por otra parte, si en efecto un traductor está autorizado para trabajar debido a sus habilidades, entonces uno se pregunta sobre la ética de lo que voy a llamar «suspensión de habilidades». El traductor suspende sus habilidades, por así decirlo, y le concede privilegios a la ideología, sea heredada o no, cuando elige una forma particular de traducir. No estoy del todo seguro de que la calidad de lo que se traduce se obtenga de esta manera.

A modo de mayor ilustración, es importante mencionar que las Sociedades Bíblicas Unidas en las Américas tomó un paso audaz cuando se emprendió la revisión de la Reina Valera 1960. Esta revisión es conocida como la Reina Valera 1995. En esta edición, Génesis 1.2 dice [...] espíritu de Dios. La decisión exegética de escribir «espíritu» sin «E» mayúscula causó mucho conflicto y debate. De hecho, se convirtió en una cuestión de mercadotecnia porque por muchos años esta revisión fue rechazada. Hoy 
en día, más y más lectores y Sociedades Bíblicas nacionales están aceptando esta edición de 1995, pero ha sido un proceso lento. Como veremos a continuación, las cuestiones de mercadotecnia y competencia también ingresan al plano de la toma de decisión ética en la labor de traducción. La NVI, publicada en 1999, por la Sociedad Bíblica Internacional se presentó como una versión que competía con la Reina Valera 1995 de la SBU. El escribir en mayúscula la palabra "espíritu" en el Antiguo Testamento le dio cierta ventaja entre las comunidades protestantes conservadoras en Latinoamérica.

\section{Exemplo 3:}

El estudio de caso que sigue presenta la situación en la cual entran en juego culturas e ideologías distintas. En un proyecto de traducción a un idioma indígena, el consultor fue confrontado por el cacique. Él había sido el que trabajó en la traducción del Nuevo Testamento que fue publicada unos treinta años antes. Aparentemente, a medida que pasó el tiempo, una teología de un Dios benevolente se desarrolló dentro de esa comunidad.

Al traducir el Antiguo Testamento y encontrar que a veces Dios era representado como un Dios celoso, o enojado, el cacique dijo que esto era inaceptable para su comunidad. Por lo tanto se rehusó a traducir estos adjetivos que describieran a Dios de una manera muy antropomórfica, porque menoscababan a Dios y la reputación de Dios sufriría extremadamente en la comunidad.

Al presentarse esta situación, ciertamente resonaban las preguntas de Chesterman: ¿cómo hemos de decidir dónde termina la responsabilidad ética del traductor/consultor — o si del todo termina? En este caso, ¿dónde termina la responsabilidad ética del consultor de traducción/ traductor? (cf. CHESTERMAN, 2001, p. 139-154). Casos como estos me hacen cuestionar el modelo ético ofrecido por Chesterman. Él desarrolla un marco teórico basado en virtudes tales como confiabilidad, veracidad, imparcialidad, y el valor para arriesgarse en el cuidado por otros. 
Él entonces sugiere que todas estas deben ser subordinadas al «saber» (CHESTERMAN, ibidem).

Pero uno se pregunta inmediatamente: ¿según el saber de quién? ¿Según el saber del cacique? ¿O según el del consultor de traducción? En cualquier caso, parece que hay otro tema que está en juego, a saber, «mejorar el texto fuente». Pym argumenta correctamente que mejorar el texto fuente yace fuera de la responsabilidad del traductor. El texto fuente debería de considerarse un fait accompli (cf. PYM, 1992, capítulo 7).

Esto sugeriría que si el texto fuente habla de un Dios celoso o un Dios que se enoja, este no debería de cambiarse o matizado. En apariencia, esto podría parecer ser una decisión o solución fácil para el consultor de traducción/traductor. Sin embargo, es un hecho conocido, que si el cacique no aprueba del proyecto de traducción y del producto final, nadie en la comunidad leerá la traducción. Así que las cuestiones de poder entran en juego con las decisiones éticas, junto con cuestiones de ideología y de mejoramiento del texto fuente. Pero quizás la pregunta más importante es, ¿queremos que el texto sea leído por la comunidad? Someto una vez más que esas preguntas siguen apareciéndose en cada esquina.

\section{Mercadotecnía y la traducción bíblica}

Una simple búsqueda en Google hecha el 21 de julio de 2015 acerca de «Mercado y traducción bíblica» ofreció 1.640 .000 resultados en 0.82 segundos. La relación entre mercadotecnia y traducción bíblica es una que la mayoría de las comunidades religiosas preferirían ignorar. Las muchas sociedades y organizaciones sin fines lucrativos que se dedican a la traducción bíblica intentan minimizar esta realidad. Además, es nuestro parecer que ni Pym (1992, capítulo 7), Chesterman (2001, p. 139-154), Towner (2004, p. 1-12), Lyotard (cf. YENGOYAN, 2003, capítulo 1), Wittgenstein (cf. YENGOUAN, ibidem) entre otros, abordan este tema explícita y abiertamente. Quizás haya algunas indicaciones presentes que se discuten, como preguntas de quién es el cliente y qué espera el cliente. Esto representa solo el principio hacia el reconocimiento de la realidad y presencia contundente que la mercadotecnia 
tiene sobre la traducción bíblica. Reconocemos que tal vez esto no sea tan relevante para los proyectos de traducción bíblica a las supuestas lenguas minoritarias, en las cuales quizás no haya una larga historia de tradición y en las cuales no existía previamente una traducción bíblica. Mientras que cuando uno está involucrado en traducir la Biblia a una lengua mayoritaria como el español para un continente con una larga tradición católica y protestante, la mercadotecnia asoma su cara una y otra vez.

Quizás el entendimiento más aceptado de mercadotecnia es aquel que sugiere que involucra el proceso de planificación y la ejecución de la concepción, fijación de precios, promoción de bienes, servicios e ideas para crear intercambios que satisfacen objetivos individuales y de las organizaciones. El asunto de satisfacer objetivos individuales y de las organizaciones parece ser la más relevante para nuestros propósitos. En otras palabras, ¿cómo se desarrolla una ética de traducción y a la vez satisface objetivos que están vinculados a los objetivos de promoción y distribución?

Cuando se lidia con traducciones bíblicas a lenguas mayoritarias, los costos son enormes y la organización sin fines lucrativos responsable por el proyecto espera que el producto satisfaga los objetivos y por ende recupere una parte - sino que es que todo - de la inversión inicial, para que de esa manera se inicien otros proyectos. La satisfacción de los objetivos se convierte en un poderoso jugador en la mesa de traducción. Como Towner ha indicado, la satisfacción puede ser buscada por más de una entidad en cualquier proyecto dado, tal como una Sociedad Bíblica nacional, alguna confesión religiosa particular, etc. (cf. TOWNER, 2004, p. 1-12).

Tal como veremos en los ejemplos que se presentan más adelante, estas y otras partes interesadas pueden ejercer una cantidad tremenda de presión sobre el traductor y el equipo de traducción. Los ejemplos que presentaremos exhiben una interesante superposición entre las presiones ideológicas y la mercadotecnia. Es nuestra intención demostrar que estos temas deben ser colocados en el primer plano de cualquier discusión o desarrollo de una ética de traducción bíblica. 


\section{Mercadotecnía: estudios de caso}

Una vez más, volveré a emplear la primera persona.

\section{Ejemplo 1 :}

Por casi diez años participé en el más reciente proyecto de la SBU de traducción al español. La meta era producir una traducción caracterizada por un lenguaje simple y contemporáneo que tuviera como su objetivo principal la comunicación de los mensajes de la Biblia. El Nuevo Testamento fue publicado en el año 2000. Esta traducción ahora se llama Traducción en lenguaje actual. Esta es verdaderamente una traducción innovadora de la Biblia, en la que conjuntos enteros del texto son reestructurados. La respuesta ha sido más que positiva. Admito que en parte muchos líderes la aceptaron porque vieron que era dirigido a niños. Siempre ha habido una actitud condescendiente hacia los niños.

La oficina de publicaciones en las Américas se puso muy feliz cuando recibió una orden por 100.000 copias del Nuevo Testamento de un obispo católico en Venezuela. Todo iba bien hasta que alguien le llamó la atención al obispo sobre la traducción de Lucas 2,7. El texto ahí dice primer hijo. Las traducciones tradicionales dicen primogénito. Esta palabra significa solo una cosa: el primer nacido. Sin embargo, no es una palabra utilizada en el lenguaje común, ni sería una palabra que la mayoría de niños entenderían. El obispo, no obstante, insistió en que usáramos la palabra histórica tradicional. ¿Por qué? Porque la palabra primogénito, para este obispo, sugería no solo que era el primer nacido, sino que también hijo único.

Sus presuposiciones ideológicas entraron en juego. Por un lado, la presión es ejercida por razones ideológicas. Por el lado de la unidad de publicaciones, la presión es impulsada por las finanzas y mercadotecnia. ¿Por qué debemos de poner en peligro una venta de 100.000 copias sencillamente por una aparentemente palabra inocente? Y esto podría conllevar a otros rechazos catastróficos. La ironía de todo esto es que el Nuevo Testamento fue cuidadosamente revisado y aprobado por el Monseñor Armando Levoratti, miembro de la Pontificia Comisión Bíblica, y fue 
publicada con una carta de aprobación por el Arzobispo de Tegucigalpa y el presidente del Consejo Episcopal Latinoamericano.

Este asunto debía ser tratado por aquellos de nosotros que somos miembros del equipo de traducción. ¿Cuáles parámetros éticos debemos de usar? Ciertamente no hay razones lingüísticas, exegéticas o de traducción para cambiar el texto. La única razón para cambiar el texto sería para satisfacer la necesidad de vender 100.000 copias. Por otra parte, compromete nuestra filosofía de traducción en producir este texto. Nuestros estudios claramente indican que la palabra "primogénito" no es una palabra comprendida fácilmente por niños.

Las preguntas siguieron surgiendo: ¿hemos de cambiar la traducción de un texto bíblico cada vez que alguien con poder de mercadotecnia solicite un cambio? ¿Llegará algún momento en la labor de la traducción bíblica cuando «ya es suficiente»? Como traductores, quizás tengamos un «voto jeronímico» al cual seguir, quizás aceptemos una ética basada en virtudes, quizás además tengamos la capacidad de distinguir claramente entre la ética personal y profesional. Pero el mensaje esencial que recibimos parece ser que cuando el poderoso señor Don Dinero habla, los traductores o se callan o se resignan.

\section{Ejemplo 2:}

La Traducción en lenguaje actual fue cuestionada por una Sociedad Bíblica nacional. El tema en cuestión fue la traducción de 1 Juan 5.1617. Como traductores discutimos el significado del texto extensamente. Después de mucha investigación, decidimos seguir lo que considerábamos que ofrecían los mejores comentarios exegéticos, incluyendo el manual de la SBU sobre las Cartas de Juan. Nuestra traducción interpreto el texto para que se refiriera a "muerte espiritual" o "muerte eterna" em vez de a simplemente "muerte", lo que es bastante ambiguo en el contexto.

Esta Sociedad Bíblica nacional envió una carta a otros Secretarios Generales de las Sociedades Bíblicas nacionales de Latinoamérica amenazando que si nosotros (los traductores) no cambiábamos la traducción de este texto en el ya publicado Nuevo Testamento, ellos no distribuirían la 
Traducción en lenguaje actual completa. Ellos exigieron a los traductores a cambiar la traducción para que el texto permaneciera ambiguo, tal como muchas traducciones tradicionales y literales.

Una vez más la decisión de cambiar un texto dado no está basada en razones exegéticas, históricas y lingüísticas. Tampoco está basada en alguna «ética superior». El cambio se origina dentro de una ideología preconcebida. Esa ideología es la que es aceptada por una mayoría de personas que están relacionadas con una Sociedad Bíblica local en particular. Esta Sociedad Bíblica nacional entonces ejerce algún tipo de presión ideológica sobre el resto de las Sociedades Bíblicas en el continente. Sin embargo, la amenaza de no distribuir la TLA produce una notable presión de mercadotecnia. Es bastante evidente que una traducción de esta magnitud requiere de una mayor inversión. Aquellos que participaron en este proyecto vinieron de diferentes regiones en Latinoamérica. Ellos también representaron distintas especializaciones. El propósito era tener un equipo interdisciplinario involucrado en todas las etapas de la traducción. Todo esto es muy costoso.

Al final, los traductores tuvimos que ceder, y nos vimos obligados a hacer un cambio en el texto debido a esta amenaza. Sacrificamos una traducción que como especialistas sentíamos que era mucho mejor debido a una presión de mercadotecnia. ¿Cuál ética, si hubo alguna, se opera aquí? ¿Hasta qué punto podemos hablar de una representación ética en el que el imperativo ético es el de representar el texto fuente? Quizás la ética de servicio se aplica más estrechamente, en el que el objetivo de la traducción está establecida por el cliente y es aceptada y negociada por el traductor. Y sin embargo, la situación aquí es un tanto diferente porque el cliente no estableció el objetivo de la traducción. El cliente en este caso recibe la traducción y luego ejerce poder sobre el produto.

En relación con este caso, se podría tener en cuenta el comentario de Pym de que una ética debería de ser capaz de abordar dilemas morales cuando surjan, pero no deberían de destacarlos innecesariamente (cf. PYM, 1992, capítulo 7). ¿Suscitó nuestra traducción de 1 Juan 5.16-17 un dilema moral innecesario? No estoy seguro de que caracterizaría la traducción como si suscitara un dilema moral. Por otra parte, nosotros como 
traductores nos enfrentamos a un dilema moral cuando somos obligados a cambiar el texto sobre la base de una amenaza de mercadotecnia.

\section{Ejemplo 3:}

Una vez más surgió un problema cuando uno de los Secretarios Generales de una Sociedad Bíblica nacional regresó de uma actividad con una iglesia de tradición pentecostal. El Secretario se alarmó por los comentarios que la gente hizo sobre la Traducción en lenguaje actual. Pronto dejó ver que su preocupación se basó primordialmente en la posibilidad de perder clientes. De hecho, él sugirió un cambio en Lucas 24.13 en donde se habla de dos seguidores de Jesús que caminaban a Emaús. En la TLA tradujimos «dos de los seguidores». El problema que surgió se basó en la interpretación de que dos seguidores quizás hayan sido una pareja; es decir, esposo y esposa. Como la palabra «seguidores» es masculina, esta traducción descartó la posibilidad de sugerir que fueran esposo y esposa.

Una vez más el asunto no era uno exegético, lingüístico o de traducción. No hay una base exegética o histórica para entender a los «dos de ellos» como esposo y esposa. Sin embargo, la posibilidad de perder clientes generó suficiente presión para que los traductores tuvieran que luchar con un posible cambio.

\section{Contribuciones positivas ofrecidas por la mercadotecnía}

Al presentar estos ejemplos, y lo que consideramos que son algunos efectos negativos de la mercadotecnia en el proceso de traducción bíblica, nos es necesario admitir que las «cuestiones de mercadotecnia» no son siempre negativas, ni tampoco es un asunto de blanco y negro. La mercadotecnia tiene el potencial de ayudar en una manera muy positiva en lo que respecta los aspectos éticos de la traducción bíblica ${ }^{1}$.

1 Quisiera darle reconocimiento y agradecimiento a mi colega Susan Mills por sus aportes con respecto a esto. Me apoyo enormemente en sus ideas en esta sección. 
No es ningún secreto que los principios de mercadotecnia nos ayudan a darnos cuenta de que todo nuestro trabajo debe tener objetivos. Estos objetivos deben ser logrados de la manera más rentable posible. Algunos sugieren que no tenemos ningún derecho moral para trabajar de otro modo. Una buena mayordomía puede ser fortalecida considerablemente por un uso adecuado de técnicas de mercadotecnia.

De hecho, el aspecto de promoción de la mercadotecnia puede ayudar a los traductores a cristalizar lo que ellos verdaderamente creen sobre sus traducciones. Además, las preguntas que ofrece la mercadotecnia pueden ayudar a los traductores a identificar sus propios puntos ciegos, y por lo tanto eliminar cargas culturales y subjetividades teológicas.

Las preguntas de mercadotecnia también pueden ayudar a los traductores a nivelar fidelidades. Los traductores de la Biblia tienden a ser extremadamente fieles al texto fuente. Los compromisos académicos y exegéticos tienen prioridad sobre todo lo demás. Las preguntas de mercadotecnia pueden ayudar a los traductores a ser fieles al cliente también. El público para quien la traducción bíblica es ofrecida merece de igual manera la fidelidad y el compromiso del traductor bíblico. Este equilibrio delicado es difícil de conseguir, especialmente cuando el público está condicionado por la tradición y otros factores no permiten que se acepte un nuevo conocimiento y traducciones superiores.

En conclusión, la mercadotecnia puede actuar como un instrumento muy útil si es introducida junto con la preocupación humanitaria y no obliga al traductor a comprometer sus importantes decisiones éticas. Si la mercadotecnia es impulsada exclusivamente por una preocupación por las ganancias, entonces se tienden a enlodar las aguas. Me parece que debe de haber siempre un lugar para la negociación, pero al mismo tiempo debe de haber lugar para los valores éticos.

\section{Hacia una ética de liberación para la traducción bíblica}

La discusión anterior ha sido articulada con el fin de servir como catalizador para pensar en la ética de la traducción bíblica. En cierto sentido, el propósito es empezar con lo más pragmático y proceder hacia la 
esfera más teórica. Es nuestra opinión que los ejemplos reales necesitan cultivar el desarrollo de cualquier marco teórico.

Nosotros dijimos al principio que nuestra intención no era desarrollar una ética rígida que proporcione un fin a la discusión. Los ejemplos ofrecidos sugieren que sería osado pensar que uno pudiera ofrecer una ética hecha y derecha que es capaz de incorporar todos los aspectos de la traducción para todas las culturas. Esto es especialmente verdadero si uno es al menos un poco escéptico de si cualquier traducción puede llegar a ser adecuadamente realizada. Lyotard quizás tenga razón cuando dice que la traducción en cualquier forma es virtualmente imposible, ya que cada lengua tiene su propio conjunto de reglas que están culturalmente determinadas y se circunscriben a una época particular (cf. YENGOYAN, 2003, capítulo 1; también LYOTARD, 1995).

Lyotard también sostiene que la traducción no solo es una tarea infinita sin cierre, pero que cada traducción engendra otra más. En otras palabras, la traducción es un proceso continuo que nunca es limpio y ordenado. Los cabos sueltos aparecen constantemente y eso forma parte de la naturaleza de la traducción. De hecho, pueda que Yengoyan tenga razón cuando afirma que "la traducción es una manera de limpieza de casa, la cual puede estar arreglada, pero la verdade belleza es mantener el desorden y el caos parcial como parte del processo" (YENGOYAN, ibidem).

La misma realidad se aplica al intento de articular una ética de traducción bíblica. La finalización y exhaustividad puede que sean una imposibilidad y, hasta cierto grado, indeseables. Nuestra propuesta para una ética de traducción bíblica comienza con la afirmación de que debido a la naturaleza del proceso mismo de traducción, será inevitablemente subjetivo, parcial y flexible, y no fingirá traer la discusión a un cierre. Quizás lo que estamos proponiendo es un tipo de imagen minimalista. Esta imagen es algo similar a lo que los antiguos griegos enseñaban mediante la elucidación de Heráclito de que uno nunca puede pisar en el mismo río otra vez. Los contextos, lenguas, culturas, ideas, cambian constantemente y esto es lo que necesita estar en el fondo de cualquier articulación teórica.

Esta imagen, como lo fue mencionado anteriormente, será siempre subjetiva y no puede aseverar ser absoluta en cualquier sentido de la palabra. Esto se debe en parte al hecho de que la traducción bíblica siempre es 
hecha en un contexto sociocultural. De todas maneras, estas realidades, en nuestra opinión, no quitan la posibilidad de sugerir una medida que pueda considerarse viable y legítima.

Empezaremos por construir sobre la sugerencia de Chesterman que las virtudes tales como la confiabilidad, veracidad, imparcialidad y el valor para arriesgarse en el cuidado por otros son cualidades válidas, humanas, que son buscadas en el desarrollo de una ética de traducción bíblica. Lo cierto es que uno tiene que estar consciente de que cada uno de estos términos necesita ser mejor definido. Por ejemplo, ¿qué es la imparcialidad? ¿Quién la define? ¿Varía de cultura en cultura la idea, concepto, y/o práctica de la imparcialidad? Pero, cualesquiera que sean las respuestas a estas preguntas, podemos estar de acuerdo con Chesterman que estas virtudes involucran las relaciones humanas. Es en este punto que quisiéramos introducir un concepto que podría ser entendido como uno que puede sostener una ética de traducción bíblica. Proponemos que una ética de traducción debería de ser una que compone, que coloca bien las cosas y a las personas, que corrige, que establece.

Spinoza habla de algo similar en términos más ontológicos (cf. DELEUZE, 2004). Tomamos la sugerencia de Spinoza y la aplicamos al traductor y al proceso de traducción, y decimos que una traducción ética es una que compone y por lo tanto empodera. Establecido en términos contrarios, cualquier traducción que disminuye, o que "desempodera", no es una traducción ética, aunque suene subjetivo. Estamos sugiriendo que este marco debe de predominar sobre los aspectos de ideología, confesión religiosa, mercadotecnia, y otros temas afines. La meta del traductor y posteriormente de la traducción debe de ser la de componer en el sentido de que coloque en forma apropiada, de establecer, de corregir, y como consecuencia, el de empoderar al individuo, a una comunidad o a una situación. El traductor debería de provocar dignidad, valía y valor mediante la traducción producida.

Nosotros afirmamos esto en categorías muy generales porque la realidad de la traducción bíblica es bastante compleja. Primero que todo, la traducción bíblica es un proceso que nunca se acaba. Es una historia sin fin que por varias razones sigue y sigue. Segundo, en nuestra experiencia la relación traductor-cliente nunca está claramente delineada. Con esto 
queremos decir que no estamos contratados directamente por aquellos que leen nuestras traducciones bíblicas. De hecho, quizás tengamos que hablar de múltiples clientes: Sociedad Bíblica nacional, líder denominacional (misionero), líderes indígenas y comunidades indígenas. Y sin embargo, ninguno de estos paga directamente nuestros salarios. Tercero, es muy diferente si estamos produciendo la única Biblia que cualquier comunidad va a leer, o si estamos produciendo una Biblia para una lengua mayoritaria. Norad es bastante contundente cuando escribe:

Ya que las Sociedades Bíblicas subvencionan la producción y venta de Biblias para ser distribuidas en áreas "pobres" del mundo, podrían establecer un monopolio y brindar la única versión de la Biblia que muchos lectores leerán o escucharán [...]. Aquellos que no son capaces de elegir estarán a la merced, por decirlo así, de cualquier teología o principio de traducción que ha impulsado la primera versión que tienen, un hecho que debería de darnos algo en qué pensar (NORAD, 2002, p. 26-27 e 30).

No queremos ser críticos del trabajo de la Sociedad Bíblica. Después de todo, es mejor que haya una Biblia a que no haya ninguna. Lo único que queremos es crear una conciencia de esta realidad cuando tratamos de articular una ética de traducción. Los asuntos de poder comercial, monopolio, y así sucesivamente no pueden ser ignorados. Es a luz de esto que sugerimos que una ética de traducción bíblica debería de buscar componer, y de esta manera empoderar al otro para existir, para tener vida.

Un componente necesario de esta ética es el servicio. Esto es bastante diferente de la ética de servicio explicado por Chesterman. Ya que estamos sugiriendo una ética para la traducción bíblica, consideramos que es legítimo sugerir un matiz más teológico para el concepto de servicio. Por esto queremos decir que los traductores y las traducciones deben de ser inmiscuidas con un espíritu de servicio en beneficio del ser humano. La traducción nunca debe de ser un fin en y para sí misma. El traductor debería de preguntar constantemente: ¿cómo puede esta traducción servir de mejor manera al supuesto cliente? En este momento no estamos preocupados por la fidelidad como lo está Chesterman, pues la fidelidad tiene el potencial de ejercer un efecto comprometedor sobre el traductor. Sin embargo estamos preocupados que un sentido profundo de servicio 
se incorpore al proceso entero de traducción.Finalmente, como parte de esta ética de traducción bíblica, proponemos que la liberación tiene que ir conjuntamente con el intento de componer, empoderar y servir (cf. DUSSEL, 2004, p. 1-166) $)^{2}$.

La traducción bíblica debería de ser un proceso en el que la liberación en su sentido más amplio se pueda lograr. Esto será ciertamente determinado contextual y culturalmente. Y, sin embargo, el intento de liberar necesita estar presente a lo largo del todo el processo de traducción bíblica.

Pues el proceso de traducción de la Biblia no debe de convertirse en una institución más, o en un subsistema más que directa o indirectamente facilita la exclusión del otro. Sugerimos que una ética de liberación es necesaria para evitar que el proceso de traducción bíblica sea una entidad de poder que coloca a la gente «afuera». Al contrario, debería de traer al «otro» hacia adentro y cultivar la vida en su mayor expresión. La ética debe buscar articular la viabilidad de un horizonte de vida, en vez de la construcción de muros de exclusión, marginalización y muerte. Una ética de liberación debe efectuarse en una acción transformadora por la que todo el conocimiento empleado en el proceso de traducción es para el desarrollo de la vida. Muchos de los proyectos de traducción bíblica son hechos en beneficio de, por el bien de y en conjunto con las comunidades de víctimas, o «comunidades victimizadas». Es por eso que una ética de liberación es tan imperativa. Una ética de liberación que brindará la oportunidad para que el proceso de traducción bíblica intervenga creativamente en el progreso cualitativo de la historia. Representa el canal mediante el cual el proceso de traducción puede transformar la espada en arado, que abrirá el camino para el desarrollo de la vida.

Una ética de liberación es una ética de responsabilidad por el otro. Podría llamarse una ética de responsabilidad radical, porque no pasará por el otro lado (no seguirá de largo) cuando sea confrontado con la víctima. La responsabilidad por el otro que es vulnerable, que está sufriendo se convierte en una prueba definitiva de una ética de la traducción bíblica. Es nuestra opinión que si la liberación está ausente de cualquier

2 Un tratamiento exhaustivo sobre la ética de liberación para un mundo globalizado. 
construcción ética, entonces llamaríamos a aquella construcción otra cosa. Dadas las realidades devastadoras que la mayoría de la gente experimenta en el planeta Tierra, una ética de traducción bíblica necesita exigir que la liberación sea un componente esencial de su marco. Afirmamos esto apasionadamente, mientras que al mismo tiempo reconocemos que es un asunto subjetivo.

\section{Consideraciones finales}

En conclusión, consideramos que estos elementos, si bien no son absolutos o exhaustivos, son necesarios para la ética de traducción bíblica, para que las cuestiones de ideología, mercadotecnia, grupos de interés, y agendas personales, puedan ser abordadas de tal manera de que ni uno solo domine el proceso de traducción. Lo que nos es claro es que esta discusión, así como la traducción bíblica, es y debe de ser un proceso sin fin. No se supone que lo que propongamos cierre este tema, sino que brinda algunos principios que en nuestra consideración son esenciales. No obstante, las preguntas permanecen. ¿Cómo abordamos éticamente una situación en la que una Sociedad Bíblica nacional decide no llevar a cabo más proyectos de traducción? ¿Existe una responsabilidad ética hacia el traductor en tal caso? ¿Cómo podemos incorporar un marco ético con justicia, y por esto nos referimos a la justicia bíblica? Estas y muchas otras preguntas aún quedan por abordarse. Son estas consideraciones que nos llevan a enfatizar que una ética de traducción bíblica debe de permanecer provisional, flexible y abierta a nuevos horizontes.

\section{Referencias:}

BRUEGGEMANN, W. Texts That Linger, Words That Explode. In: Theology Today vol. 54, n. 2, 1997, p. 180-199.

CHERTERMANN, A. Proposal for a Hieronymic Oath. In: The Translator, vol. 7, n. 2, 2001, p. 139-154. 
VOTH, E.

DELEUZE, G. En Medio de Spínoza. Buenos Aires: Ediciones Cactus, 2004.

DUSSEL, E. Ética de la liberación en la edad de la globalización y de la exclusión. Madrid: Editorial Trotta, 2004.

LYOTARD, J.-F. La condición postmoderna. Buenos Aires: Red Editorial Iberoamericana, 1995.

NORAD, S. New and Familiar: The Dynamics of Bible Translation. In: BRENNER, A.; VAN HENTEN, J.W. (Eds.). Bible Translation on the Threshold of the TwentyFirst Century. Sheffield: Sheffield Academic Press, 2002, p. 26-30.

PORTER, S. The Contemporary English Version and the Ideology of Translation. In: PORTER, S.; HESS, R. (Eds.). Translating the Bible: Problems and Prospects. Sheffield: Sheffield Academic Press, 1999.

PYM, A. Translation and Text Transfer. New York: Perter Lang, 1992. En la obra, especialmente el Capítulo 7.

TOWNER, P. Ethics and Bible Translation: A Working Paper. Unpublished paper presented in Rome, April 2004, p. 1-12.

VOTH, Steven. Righteousness and/or Justice: A Contextualized Analysis of "tsedeq" in the KJV (English) and the RVR (Spanish). In: VOTH, S; SCORGIE, G.; STRAUSS, M. (eds.). The Challenge of Bible Translation. Grand Rapids: Zondervan, 2003. En la obra, especialmente el Capítulo 14.

YENGOYAN, A. Lyotard and Wittgenstein and the Question of Translation. In: RUBEL, P.; ROSMAN, A. (Eds.). Translating Cultures. Oxford: Berg, 2003. En la obra, especialmente el Capítulo 1.

Recebido: 27/11/2015

Received: 11/27/2015

Aprovado: 15/01/2016

Approved: 01/15/2016 